\title{
Compatibility of ionic liquids with hydraulic system components
}

\author{
Kambič, M. ${ }^{a}{ }^{,}$, Kalb, R. ${ }^{b}$, Tič, V. ${ }^{c}$, Lovrec, D. ${ }^{c}$ \\ ${ }^{a}$ Olma d.o.o., Ljubljana, Slovenia \\ ${ }^{\mathrm{b}}$ Proionic GmbH, Grambach, Austria \\ 'University of Maribor, Faculty of Mechanical Engineering, Maribor, Slovenia
}

\begin{abstract}
A B S T R A C T
The aim of this work was to identify, which of the known ionic liquids used within the technical area, primarily as a lubricant, would also be appropriate for use as a hydraulic fluid. In this context, their suitability has been proved based on experimental research with respect to the appropriate physical and chemical properties as required for mineral based hydraulic fluid. Primary aim of the research was to determine the ability of ILs to protect against corrosion, which is one of the important factors in choosing an ionic liquid. The results show that, despite excellent lubricating properties, certain ILs fail on this corrosion test. Except the corrosion protection performance of the basic hydraulic components parts, e.g. hydraulic pumps and valves, in the foreground was their compatibility with other materials used within other parts of hydraulic system, e.g. coating of the hydraulic tank and the filter material. For this purpose standard tests methods for mineral based hydraulic oils have been used, supplemented with non-standard tests, carried out at the same conditions as they occur during the operation of the hydraulic system.
\end{abstract}

\section{ARTICLE INFO}

Keywords:

Ionic liquids;

Hydraulic fluid;

Corrosion protection;

Material compatibility

*Corresponding author:

milan.kambic@olma.si

(Kambič, M.)

Article history:

Received 13 June 2018

Revised 25 November 2018

Accepted 29 November 2018

\section{References}

[1] Majdič, F., Kalin, M. (2014). Test rig and comparison of pressure changes at transient phenomena in water- and oil-based power-control hydraulics, Journal of Vibroengineering, Vol. 16, 401-411.

[2] Majdič, F., Kalin, M. (2014). Characteristics of the stationary behaviour of water- and oil-based power-control hydraulics, Mechanika, Vol. 20, No. 3, 274-281, doi: 10.5755/j01.mech.20.3.5301.

[3] Majdič, F., Velkavrh, I., Kalin, M. (2013). Improving the performance of a proportional 4/3 water-hydraulic valve by using a diamond-like-carbon coating, Wear, Vol. 297, No. 1-2, 1016-1024, doi: 10.1016/j.wear.2012.11.060.

[4] Kalb, R. (2015). Ionic liquids - A new generation of high-tech engineering liquids, In: Proceedings of international conference Fluid Power 2015, University of Maribor, Slovenia, 49-77.

[5] Zhou, F., Liang, Y., Liu, W. (2009). Ionic liquid lubricants: Designed chemistry for engineering applications, Chemical Society Reviews, Vol. 9, No. 38, 2590-2599, doi: 10.1039/b817899m.

[6] Kondo, Y., Yagi, S., Koyama, T., Tsuboi, R., Sasaki, S. (2012). Lubricity and corrosiveness of ionic liquids for steelon-steel sliding contacts, Proceedings of the Institution of Mechanical Engineers, Part J: Journal of Engineering Tribology, Vol. 226, No. 11, 991-1006, doi:10.1177/1350650112456127.

[7] Jiang, D., Hu, L., Feng, D. (2011). Crown-type ionic liquids as lubricants for steel-on-steel system, Tribology Letters, Vol. 41, No. 2, 417-424, doi: 10.1007/s11249-010-9726-x.

[8] Fox, M.F., Priest, M. (2008). Tribological properties of ionic liquids as lubricants and additives, Part 1: Synergistic tribofilm formation between ionic liquids and tricresyl phosphate, Proceedings of the Institution of Mechanical Engineers, Part J: Journal of Engineering Tribology, Vol. 222, No. 3, 291-303, doi: 10.1243/13506501JET387.

[9] Amiril, S.A.S., Rahim, E.A., Syahrullail, S. (2017). A review on ionic liquids as sustainable lubricants in manufacturing and engineering: Recent research, performance, and applications, Journal of Cleaner Production, Vol. 168, 1571-1589, doi: 10.1016/i.jclepro.2017.03.197. 
[10] Regueira, T., Lugo, L., Fernández, J. (2014). Ionic liquids as hydraulic fluids: Comparision of several properties with those of conventional oils, Lubrication Science, Vol. 26, No. 7-8, 488-499, doi: 10.1002/ls.1235.

[11] Nyberg, E., Respatiningsih, C.Y., Minami, I. (2017). Molecular design of advanced lubricant base fluids: Hydrocarbon-mimicking ionic liquids, RSC Advances, Vol. 7, No. 11, 6364-6373, doi: 10.1039/c6ra27065d.

[12] Kambic, M., Kalb, R., Tasner, T., Lovrec, D. (2014). High bulk modulus of ionic liquid and effects on performance of hydraulic system, The Scientific World Journal, Vol. 2014, Article ID 504762, doi: 10.1155/2014/504762.

[13] Pensado, A.S., Comuñas, M.J.P., Fernández, J. (2008). The pressure-viscosity coefficient of several ionic liquids, Tribology Letters, Vol. 31, No. 2, 107-118, doi: 10.1007/s11249-008-9343-0.

[14] Minami, I. (2009). Ionic liquids in tribology, Molecules, Vol. 14, No. 6, 2286-2305, doi: 10.3390/molecules 1406 2286.

[15] Van Rensselar, J. (2010). Cover story: Unleashing the potential of ionic liquids, Tribology and Lubrication Technology, Vol. 66, No. 4, 24-31.

[16] Ye, C., Liu, W., Chen, Y., Yu, L. (2001). Room-temperature ionic liquids: A novel versatile lubricant, Chemical Communications, No. 21, 2244-2245, doi: 10.1039/b106935g.

[17] Jiménez, A.-E., Bermúdez, M.-D. (2007). Ionic liquids as lubricants for steel-aluminium contacts at low and elevated temperatures, Tribology Letters, Vol. 26, No. 1, 53-60, doi: 10.1007/s11249-006-9182-9.

[18] Pejaković, V., Kronberger, M., Mahrova, M., Vilas, M., Tojo, E., Kalin, M. (2012). Pyrrollidinum sulfate and ammonium sulfate ionic liquids as lubricant additives for steel/steel contact lubrication, Proceedings of the Institution of Mechanical Engineers, Part J: Journal of Engineering Tribology, Vol. 226, No. 11, 923-932, doi: 10.1177/1350 650112448978

[19] Kambić, M., Kalb, R., Lovrec, D. (2015). Lubrication properties of ionic liquids suitable for use within hydraulic systems, In: Proceedings of International Conference Fluid Power 2015, University of Maribor, Slovenia, 79-93.

[20] Uerdingen, M., Treber, C., Balser, M., Schmitt, G., Werner, C. (2005). Corrosion behaviour of ionic liquids, Green Chemistry, Vol. 7, No. 5, 321-325, doi: 10.1039/b419320m.

[21] Standard DIN 51386-1 (1986). Testing of corrosion preventive oils in a condensation water alternating atmosphere.

[22] Standard ISO 6270-2:2005 (2005). Paints and varnishes - Determination of resistance to humidity - Part 2: Procedure for exposing test specimens in condensation-water atmospheres.

[23] Standard ASTM D130-12 (2012). Standard test method for corrosiveness to copper from petroleum products by copper strip test.

[24] Kambič, M., Kalb, R. (2013). Corrosion and lubrication properties of some lonic liquids, Ventil, Vol. 19, No. 5, 364368.

[25] Lovrec, D. (2017). Ionic liquid as a novel, high performance hydraulic fluid - Selection process, In: ISCAME 2017: Proceedings, $5^{\text {th }}$ International Scientific Conference on Advances in Mechanical Engineering, University of Debrecen, Hungary, 300-305.

[26] Standard ISO 13357-2:2005 (2005). Petroleum products - Determination of the filterability of lubricating oils Part 2: Procedure for dry oils. 


\section{APEM}

\title{
Kompatibilnost ionskih tekočin s hidravličnimi komponentami
}

\author{
Kambič, M. ${ }^{\mathrm{a},{ }^{*}}$, Kalb, R. ${ }^{\mathrm{b}}$, Tič, ${ }^{\mathrm{c}}{ }^{\mathrm{c}}$, Lovrec, D. ${ }^{\mathrm{c}}$ \\ aOlma d.o.o., Ljubljana, Slovenia \\ ${ }^{\mathrm{b}}$ Proionic GmbH, Grambach, Austria \\ 'University of Maribor, Faculty of Mechanical Engineering, Maribor, Slovenia
}

\section{POVZETEK}

Namen raziskave je bil ugotoviti, katere izmed znanih ionskih tekočin (IL), ki se v tehniki uporabljajo predvsem kot mazivo, bi bile primerne tudi za uporabo kot hidravlične tekočine. Ustreznost IL se je dokazala na podlagi eksperimentalnih raziskav fizikalnih in kemijskih lastnosti, ki so merodajne za hidravlične tekočine na osnovi mineralov. Osnovni cilj raziskave je bil ugotoviti sposobnost IL-jev za zaščito pred korozijo, kar je eden izmed pomembnih dejavnikov pri izbiri ionske tekočine. Rezultati kažejo, da se nekatere IL kljub odličnim mazalnim lastnostim na korozijskem preizkusu izkažejo za neprimerne. Razen korozijske zaščite osnovnih hidravličnih delov, npr. hidravlične črpalke in ventili, je v ospredju raziskave tudi združljivost IL-jev z materiali, ki so uporabljeni $\mathrm{v}$ drugih delih hidravličnega sistema, npr. premaz hidravličnega rezervoarja in filtrirni material. $V$ ta namen so bile uporabljene standardne preizkusne metode namenjene testiranju mineralnih olj, dopolnjene z nestandardnimi preizkusi, izvedenimi pri enakih pogojih, kot se pojavijo pri delovanju hidravličnega sistema.

\section{PODATKI O ČLANKU}

Ključne besede: Ionske tekočine; Hidravlične tekočine; Korozijska zaščita; Kompatibilnost materialov

*Kontaktna oseba: milan.kambic@olma.si (Kambič, M.)

Zgodovina članka:

Prejet 13. junija 2018

Popravljen 25. novembra 2018

Sprejet 29. novembra 2018 